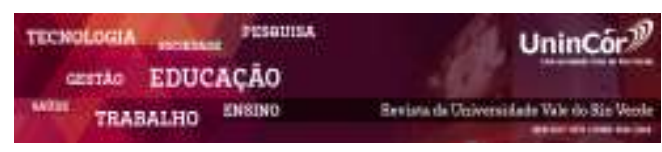

Revista da Universidade Vale do Rio Verde ISSN: 1517-0276 / EISSN: 2236-5362 Vol. 16 | n. 1 | Ano 2018

Bruna Wood Almeida Mendes Universidade José do Rosário Vellano

(UNIFENAS)

bruninhawood@gmail.com

Renata Alves de Lima

Universidade José do Rosário Vellano

(UNIFENAS)

renatalimanovais@gmail.com

Sildilaine Aparecida Correia Costa Universidade José do Rosário Vellano

(UNIFENAS)

sildilaine0302@gmail.com

Giuliano Roberto da Silva

Universidade José do Rosário Vellano

(UNIFENAS)

Faculdade Presbiteriana Gammon (FAGAMMON) Centro Mineiro de Ensino Superior (CEMES) giumusc@gmail.com

Gerusa Dias Siqueira Vilela Terra Universidade José do Rosário Vellano (UNIFENAS)

gerusa.terra@unifenas.br

Cassiano Merussi Neiva

Universidade Estadual de São Paulo (UNESP)

cassiano.neiva@unifenas.br

Bruno Barbosa Rosa Universidade José do Rosário Vellano (UNIFENAS)

brunobarbosa1991@yahoo.com.br

\section{TREINAMENTO DE FORÇA E CONCORRENTE EM ADOLESCENTES COM SOBREPESO INTEGRANTES DO PROJETO GERAÇÃO SAÚDE}

\section{RESUMO}

Entre as alterações associadas à obesidade, a resistência insulínica e intolerância a glicose, a diabetes mellitus (DM), a hipertensão arterial sistêmica (HAS) e as dislipidemias podem ser notadas como mais comuns, caracterizando um estado precursor para a Síndrome Metabólica. Estudos demonstram efeitos benéficos do exercício de força e concorrente para a saúde das pessoas, e, principalmente na pré e adolescência, auxiliando na melhora das qualidades físicas, melhora postural, fortalecimento dos ossos e articulações, adaptações cardiovasculares, diminuição do percentual de gordura, melhora das concentrações de colesterol total, HDL, LDL, VLDL e controle da pressão arterial sistêmica. O objetivo do estudo foi observar as respostas do treinamento de força e concorrente em adolescentes obesos após 12 semanas de treinamento prático com quatro sessões de exercício de força e concorrente durante a semana. Para tal, foram estudados indivíduos de ambos os gêneros entre os 12 e 19 anos de idade com IMC elevado sem experiência no treinamento orientado. Após um período de adaptação a série de exercícios, foi determinada uma zona alvo de treinamento após a realização de um teste de carga máxima em cada exercício, os exercícios escolhidos são os seguintes: (Supino Reto, Pulley Costas, Cadeira Extensora, Mesa Flexora, Rosca Direta, Rosca Tríceps, Leg Press, Remada Alta e Crunch de Abdômen). No trabalho concorrente foi executado além do trabalho de força citado anteriormente, uma caminhada rápida na esteira elétrica em uma intensidade de $75 \%$ da frequência cardíaca máxima com duração de 30 minutos. Logo, percebeu-se uma mudança significativa na variável FC em ambos os grupos (masculino e feminino) no treinamento concorrente, e também na variável PAS no grupo força, ao serem comparados ao grupo controle, porém, em relação aos indicadores antropométricos (Massa Corporal Total, IMC e Percentual Geral de Gordura) e PAD, não houve uma mudança satisfatória, provavelmente devido ao fato desses adolescentes não terem tido um acompanhamento nutricional.

Palavras-chave: Treinamento de Força. Treinamento Concorrente. Sobrepeso.

\section{STRENGTH AND COMPETITOR TRAINING IN ADOLESCENTS WITH OVERWEIGHT INTEGRATION OF THE GENERATION HEALTH PROJECT}


common, featuring a precursor state to the metabolic syndrome. Studies have shown beneficial effects of strength training and competing for people's health, and especially in pre and adolescence, assisting in the improvement of physical qualities, postural improvement, strengthening of bones and joints, cardiovascular changes, decrease the percentage of fat, improves of total cholesterol, HDL, LDL, VLDL and control of blood pressure. The aim of the study was to observe the responses of strength training and competing in obese adolescents after 12 weeks of practical training with four strength training sessions and competing during the week. To this end, individuals of both genders were studied between 12 and 19 years of age with high BMI without experience-oriented training. After a period of adaptation to exercise series, was given a training target zone after the completion of a full load test each year, the following chosen exercises are: (Supine Straight, Pulley Back, Stretcher Chair, Flexor Table, Direct thread, thread Triceps, Leg Press, High Remada and Crunch Abdomen). In concurrent work was performed in addition to the workforce previously mentioned, a quick walk on the treadmill at an intensity of $75 \%$ of maximum heart rate lasting 30 minutes. Therefore, a significant change was noticed in the FC variable in both groups (male and female) in the concurrent training, and also the PAS variable in strength group, when compared to the control group, however, in relation to anthropometric indicators (body mass overall, BMI and Fat percentage General) and DBP, there was no satisfactory change, probably due to the fact that these teenagers have not had a nutritional monitoring.

Keywords: Strength training. Competitor training. Overweight.

Recebido em: 17/10/2017 - Aprovado em: 18/01/2018 - Disponibilizado em: 15/07/2018

\section{INTRODUÇÃO}

Atualmente um dos principais problemas de saúde pública enfrentados pela sociedade é a obesidade. A Pesquisa de Orçamentos Familiares (POF 2008-2009) mostrou que 21,7\% dos meninos e 19,4\% das meninas prevaleceram em um quadro de sobrepeso, e 5,9\% e 4,0\% dos adolescentes brasileiros de 10 a 19 anos com obesidade (IBGE, 2010).

É preocupante o aumento da obesidade infanto juvenil tendo relação com várias comorbidades, sendo uma delas as doenças cardiovasculares. Sendo também associado com a diabetes mellito tipo 2 , acidente vascular cerebral, hipertensão arterial sistêmica, dislipidemias e infarto agudo do miocárdio (WHO, 2000), a obesidade infantil pode estar envolvida em consequências socias e psicológicas, podendo afetar a auto estima do jovem obeso, acarretando consequências por um longo período de tempo (ABRANTES; LAMOUNIER \& COLSIMO 2002). No Brasil, as taxas de adolescentes acima do peso ideal seguiram o mesmo nível nas últimas duas décadas, com prevalência de 7,7\% nos anos1980, para ambos os sexos (OGDEN \& CARROLL e FLEGAL, 2008). Essa taxa já atingiu $17,9 \%$ para o sexo masculino e $15,4 \%$ para o sexo feminino na última pesquisa nacional em 2002-2003 (NEUTZLING et al., 2000). 
Observa- se que há um crescente aumento de várias disfunções crônico-degenerativo em diferentes populações, especialmente a Síndrome Metabólica (SM) (CARMO et al, 2003). A SM, que recebe diversos nomes como, por exemplo, síndrome plurimetabólica ou ainda síndrome $\mathrm{X}$, é a incidência conjunta de algumas doenças ou desordens metabólicas como a obesidade, dislipidemias, hipertensão arterial sistêmica (HAS), resistência insulínica e diabetes, nas quais a obesidade, além de ser uma delas, parece contribuir para o desenvolvimento de doenças crônicas (THOMAS et al., 2007).

Durante anos a composição corporal e a manutenção estável do peso apresenta um resultado de um balanço fidedigno entre a ingestão e o gasto calórico (SCHUTZ, 1995); qualquer diferença nesta relação desenvolve o processo da obesidade (DIETZ \& GORTMAKER, 1985; BOUCHARD \& TREMBLAY, 1990). Sem dúvida um dos principais fatores da obesidade são os distúrbios alimentares e a falta de atividade física (RUIZ \& RUIZ 1993; SCHUTZY, 1995).

A prática de exercícios físicos, sejam eles aeróbios ou anaeróbios, com a orientação de um profissional indicam, através de estudos, inúmeros benefícios para a saúde do praticante sob diversos aspectos no organismo. Além de auxiliar na melhora das qualidades físicas, postura, fortalecimento dos ossos e articulações, adaptações cardiovasculares, diminuição do percentual de gordura, melhora das concentrações de colesterol total, HDL, LDL, VLDL e controle da pressão arterial sistêmica (NELSON et al., 2007).
O objetivo do estudo foi analisar a influência de um protocolo de treinamento de força e concorrente no excesso de gordura corporal em adolescentes com indicadores da síndrome metabólica integrantes do projeto geração saúde.

\section{MÉTODOS}

Participaram do estudo, adolescentes integrantes do Projeto Geração Saúde da cidade de Alfenas - MG, com indicativos de SM, segundo os critérios da "IDF e National Cholesterol Education Program's Adult Treatment Panel III", e sedentários (ausência de exercício físico regular) (IDF, 2001).

Para a realização da prática do estudo, foram avaliados 136 estudantes das escolas públicas e selecionados 32 indivíduos jovens entre 12 e 19 anos completos, de ambos os sexos. Foram então, encaminhados à academia cadastrada do município, para iniciarem os protocolos de treinamento após a leitura e assinatura do Termo de Consentimento Livre e Esclarecido (TCLE).

Os sujeitos do estudo foram submetidos a avaliações antropométricas na fase pré e pós treinos com o objetivo de aferição dos indicadores antropométricos. Para tanto, foram avaliados: i) Massa Corporal Total (MCT): através do uso de "Balança para Pesagem Humana", da marca Filizzola; ii) Estatura: através do uso de aparelho "Estadiômetro Compacto Tipo Trena", da marca Sanny; iii) Índice de Massa Corporal (IMC): calculado com base na fórmula: peso/altura ${ }^{2}$ 
(CASTRO, MATO \& GOMES, 2006); iv) Circunferência de Cintura (CC): mensurada em $\mathrm{cm}$, com uma fita métrica, com precisão de 0,1 $\mathrm{cm}$, aplicada acima da crista ilíaca, paralela ao solo, com o indivíduo em pé, o abdome relaxado, braços ao longo do corpo e os pés unidos; v) Relação Cintura Quadril (RCQ): medida na extensão máxima das nádegas, em centímetros, com uma trena flexível e inextensível, com resolução de $0,1 \mathrm{~cm}$, sendo a mesma colocada com firmeza, sem esticar excessivamente, evitando-se assim a compressão do tecido subcutâneo (FERREIRA et al., 2006). A RCQ foi calculada com a CC $(\mathrm{cm})$ dividida pela circunferência do quadril (CQ) (cm) (GHARAKHANLOU et al., 2012). Foram adotados os pontos de corte propostos por Yusuf et al. (2005), valores $\geq 1,0$ para homens $\mathrm{e} \geq 0,80$ para mulheres, sendo considerados riscos cardiovasculares; vi) Percentual de Gordura (\%G): foi utilizada a fórmula de Siri (1961), em que $\%$ Gord $=(495 / \mathrm{D})-450$.

Também foram realizadas avaliações hemodinâmicas nas fases pré e pós treinos dos seguintes indicadores: Pressão Arterial Sistólica (PAS), Pressão Arterial Diastólica (PAD) e Frequência Cardíaca de Repouso (FCR). A PAS/PAD, assim como a FCR será mensurada no braço direito apoiado ao nível do coração, com o tamanho do manguito apropriado ao perímetro do braço do indivíduo. Foi considerado com HAS o indivíduo com valores de PA sistólica/diastólica $\geq$ 140/90 mmHg, respectivamente (IDF, 2001).

Após um período de adaptação a série de exercícios foi determinada uma zona alvo de intensidade de treinamento, após a realização de um teste de repetições máximas de carga em cada exercício (BAECHE \& EARLE, 2010). Os exercícios escolhidos foram os seguintes: (Supino Reto, Pulley Costas, Cadeira Extensora, Cadeira Flexora, Rosca Direta, Tríceps Testa, Leg Press, Remada Alta, Panturrilha no Leg Press e Abdominal). Para a realização deste sistema, os grupos realizaram quatro séries de trabalho de força localizado abordando todos os grupos musculares, em periodicidade de quatro sessões de treinamento na semana, além do treinamento aeróbico (caminhada intensa na esteira elétrica). O período total de treinamento foi de 12 semanas, quando os participantes foram reavaliados, após o protocolo de exercícios físicos, havendo uma comparação entre pré-treino para o pós-treino.

Os voluntários selecionados foram divididos em três grupos de participantes e distribuídos aleatoriamente da seguinte forma: Grupo de treino de força (GTF) $(n=6)$ : realizou quatro séries de dez repetições com cargas na intensidade de $85 \%$ da carga máxima e intervalo entre as séries entre 2 minutos (BITTENCOURT, 1986); Grupo de treino concorrente (GTC) $(n=14)$ : realizou o treinamento aeróbio sendo este de 30 minutos de caminhada intensa na esteira elétrica na zona alvo de treino de $70 \%$ da frequência cardíaca máxima e, posteriormente, o treinamento de força com cargas na intensidade $75 \%$ da carga máxima, sendo que este sofre uma alteração para três séries de 10 repetições com intervalo de 1 minuto e 30 segundos (FAIGENBAUM \& WESTCOYY, 2009) juntamente ao treinamento de força; Grupo Controle Destreinado (GCD) ( $n=12)$ : foi avaliado nos testes físicos, porém, foi sugestionado a eles que continuassem suas atividades cotidianas 
normais, não sofrendo nenhuma intervenção de atividades físicas.

Todo e qualquer procedimento do presente estudo, só teve início após a aprovação do presente projeto, pelo CONEP, estando o presente estudo em plena consonância com as normas éticas da resolução CNS 466 de 2012 para estudos envolvendo Seres Humanos, bem como as diretrizes da Declaração de Direitos Humanos de Helsinki. O projeto foi encaminhado ao Comitê de Ética em Pesquisa da instituição, sendo aprovado com $\mathrm{n}^{\mathrm{o}}$ de parecer: 1.308 .322 .

Ao término das coletas de dados, os mesmos foram submetidos à verificação da existência ou não de parametria pela aplicação de teste de normalidade. Após verificação do estado de parametria dos dados dos grupos, os mesmos foram submetidos a teste de comparação estatística conforme apropriado. Todo tratamento estatístico foi realizado através do uso programa de análise estatística, SPSS v.17.1 e o grau de significância adotado em todos os casos foi para um valor de $\mathrm{p} \leq 0,05$.

\section{RESULTADOS E DISCUSSÃO}

Nota-se que na massa corporal (Figura 1), em ambos os grupos não houve mudanças estatísticas significativas em sua comparação (pré e pós-treinamento). Estes tipos de treinamento evidenciam a influência do treinamento resistido na composição corporal (SOTHERN et al., 2000) e na densidade mineral óssea (MORRIS et al., 1997).
Figura 1 - Massa corporal pré e pós-intervenção.

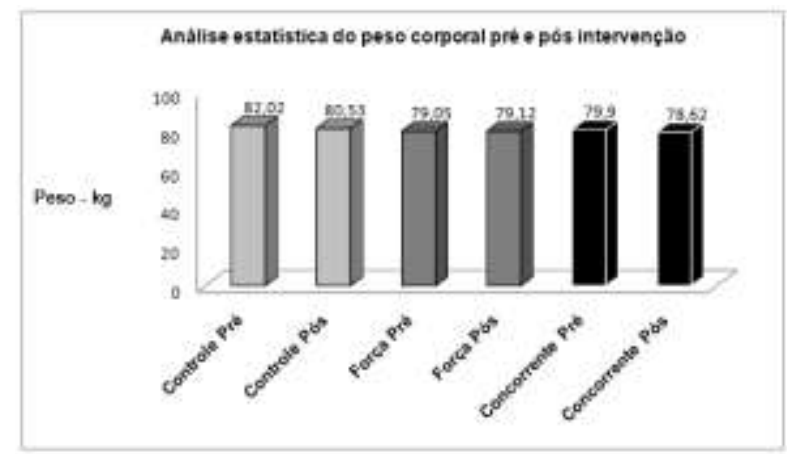

Fonte: Dados da pesquisa realizada pelos autores.

Em relação ao IMC (Figura 2), quando observado e comparado estatisticamente os valores pré e pós de ambos os grupos, e em relação ao grupo controle nota-se que não houve mudança significativa.

Figura 2 - IMC pré e pós-intervenção.

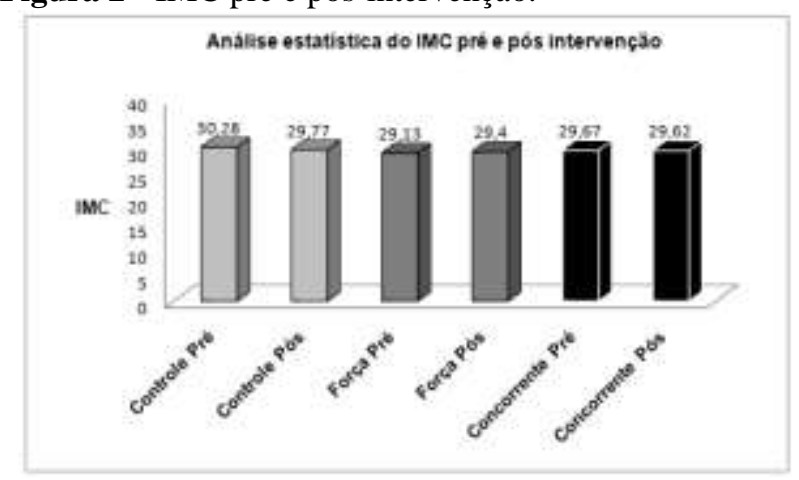

Fonte: Dados da pesquisa realizada pelos autores.

Estes resultados corroboram com os achados de Porto et al. (2013), onde desenvolveu um protocolo de treinamento com nove crianças com média de idade de dez anos, obesas e sedentárias. O protocolo estabelecido consistiu em dez semanas de treinamento de força, desenvolvida três vezes por semana, com sessões de 50 minutos cada. $O$ treinamento foi dividido em aquecimento em 10 minutos, exercícios com pesos durante 30 minutos e relaxamento durante 10 minutos Após o término, não ocorreu diferença 
significativa entre os valores pré e pósintervenção.

Em se tratando da RCQ, demonstrada pela Figura 3, a pesquisa atual com metodologia força e concorrente, na qual não houve atuação nutricional, observou coerência entre os resultados quando comparados com o estudo de Barbosa Filho et al. (2011), visto que ambos não tiveram diferenças entre os valores de RCQ pré e pós-treinamento e nem acompanhamento nutricional.

Figura 3 - RCQ pré e pós-intervenção.

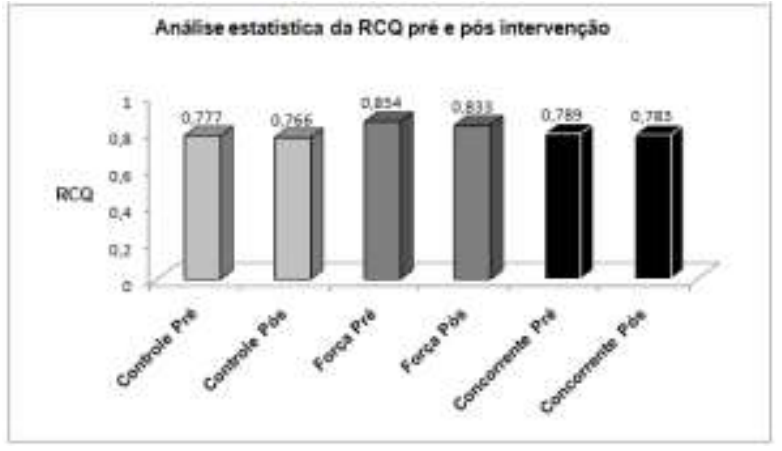

Fonte: Dados da pesquisa realizada pelos autores.

Como mostra a Figura 4 houve redução significativa entre os valores aferidos de PAS entre o grupo Força pós com o grupo Controle pré, mantendo inalterada em relação aos demais grupos. A pesquisa mostra que após o programa de treinamento de força houve uma redução significativamente, no aspecto hemodinâmico, representado pela PAS, possivelmente acompanhado de alterações endoteliais e presença de liberação de óxido nítrico, como fator cardioprotetor em resposta ao treinamento. Segundo Garcia \& Incerpi (2008), durante o período de sobrecarga vascular induzida pelo treinamento, há liberação endotelial de óxido nítrico, responsável pela vasodilatação periférica, e também associados a fatores cardioprotetores, com melhora da circulação coronariana e hipotensão pós treinamento.

Figura 4 - PAS pré e pós-intervenção.

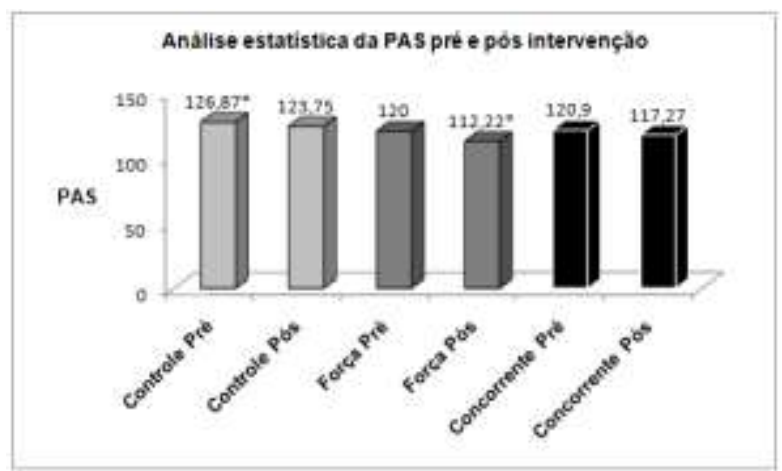

Fonte: Dados da pesquisa realizada pelos autores.

No estudo de intervenção multidisciplinar de Silva, Bianchini e Nardo Júnior (2013), foram avaliados adolescentes com idade entre 10 e 18 anos, que executaram um protocolo prático para grupos musculares localizados como, por exemplo, abdominais, flexão de braço, agachamento e exercícios com a medicine ball com duração de $20 \%$ do tempo de intervenção, e também foi realizada caminhada/corrida durante $30 \%$ do tempo restante de intervenção e no restante do tempo $(50 \%)$ foi realizado basquetebol. $\mathrm{O}$ estudo teve duração de 16 semanas com uma frequência de três vezes semanais com duração de uma hora por sessão. Durante todo o protocolo houve acompanhamento nutricional e pediátrico dos adolescentes tendo como resultado a melhora dos valores aferidos de PAS alterando de $119,50 \pm 10,06$ inicial para $114,50 \pm 11,15$ final.

Quando observado a figura 5 comprovase que não ocorreu nenhuma mudança significativa em relação aos valores de PAD após a execução do protocolo prático, o que corrobora 
com o trabalho desenvolvido por Martinelli et al. (2010), onde avaliou 20 indivíduos com hipertensão arterial, para análise de respostas fisiológicas do treinamento. $\mathrm{O}$ período experimental se deu em 16 semanas de treinamento aeróbico, com duração de 40 minutos cada sessão. Após, não foi observado mudanças estatisticamente significativas entre os grupos avaliados.

Figura 5 - PAD pré e pós-intervenção.

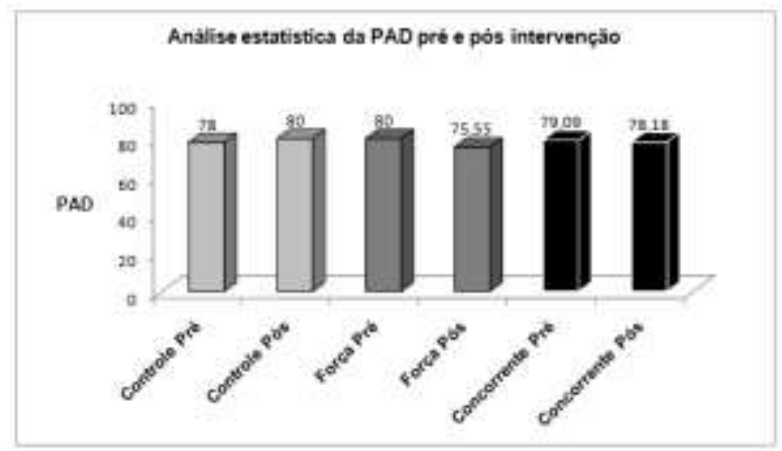

Fonte: Dados da pesquisa realizada pelos autores.

Ao analisar os resultados da FC, pode-se notar que esta apresentou mudanças significativas quando comparado o grupo concorrente pós em relação ao grupo controle pós e sem nenhuma mudança em relação aos demais grupos (Figura $6)$.

Em um estudo de Sabia, Santos \& Ribeiro (2004), o qual conteve um protocolo de treinamento executado por adolescentes, com duração de 16 semanas e uma frequência semanal de três vezes, os voluntários foram divididos aleatoriamente em dois grupos, o primeiro realizou atividade aeróbia, no qual foi aplicado um treinamento aeróbio com duração de 60 minutos de caminhada contínua, e o segundo grupo realizou atividades anaeróbias, no qual foi aplicado o protocolo intervalado caracterizado por atividades físicas com duração de 60 minutos, onde o exercício proposto foi uma corrida de alta intensidade contínua e circuito com pesos. Ao final da execução dos protocolos pode verificar que a FC diminuiu para ambos os grupos, mas somente no grupo dois houve uma mudança significativa $(p=0,004)$.

Figura 6 - FCR pré e pós-intervenção.

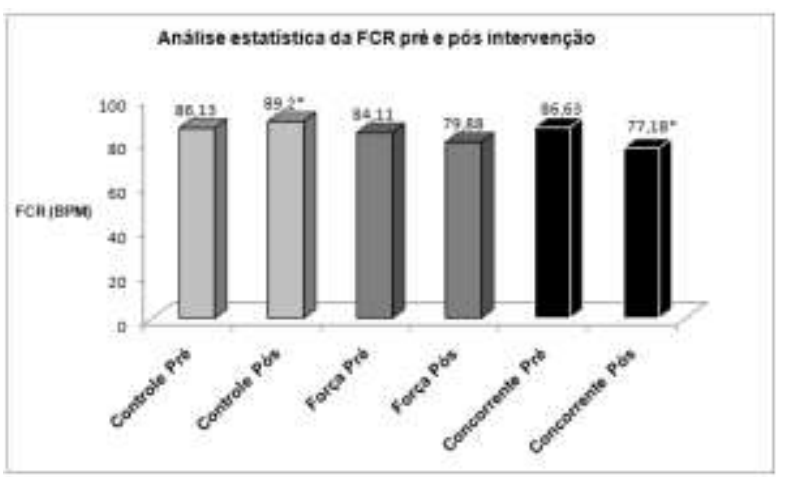

Fonte: Dados da pesquisa realizada pelos autores.

Ao observar a Figura 7, nota-se que os resultados do percentual de gordura quando comparados aos grupos pré e pós de ambos os protocolos, e o grupo controle, ao final da intervenção prática, não ocorreu nenhuma modificação significativa.

Figura 7 - \%G pré e pós-intervenção.

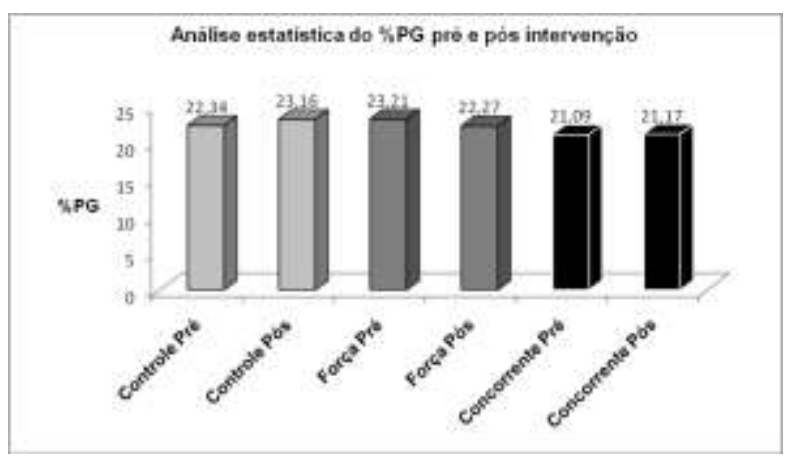

Fonte: Dados da pesquisa realizada pelos autores.

Enquanto no estudo de FARIAS et al. (2009) que elaborou um estudo comporto por 
adolescentes com idade entre 10 e 15 anos que foram divididos em dois grupos: caso e controle. O grupo controle foi orientado a participar das aulas de educação física convencionais da escola com duração de 60 minutos cada aula, estas eram compostas por atividades como recreação e jogos. O grupo caso, além das aulas de Educação Física escolar foram submetidos a atividades físicas programadas e com monitoração da FCMáx. Ao final da intervenção pode constatar uma melhora de forma significativa no percentual de gordura do grupo caso no pré-teste para o pós-teste (p < 0,01) em ambos os sexos.

\section{CONCLUSÃO}

Conclui-se que após a aplicação do protocolo proposto, observou-se que nos índices \% de Gordura, PAD, IMC e RCQ, não houve mudanças estatísticas em relação ao quadro inicial, porém na $\mathrm{FC}$ e $\mathrm{PAS}$, houve redução significativa entre o grupo concorrente pós e pré, mostrando efeito benéfico na atividade cronotrópica e inotrópica cardíaca, obtendo assim melhora na eficiência contrátil do miocárdio. Apesar de algumas mudanças benéficas do treinamento, vale salientar que os resultados poderiam ser ainda melhores caso houvesse acompanhamento nutricional adequado, como já comprovado em diversos trabalhos, a associação entre os fatores, atividade física juntamente ao controle alimentar.

\section{REFERÊNCIAS}

Abrantes, M. M., Lamounier, J. A., Colsimo, E. A. Prevalência de sobrepeso e obesidade nas regiões sudeste e nordeste. J Pediatr. Rio J. 2002; 78: 335-40.
Baeche, T. R., Earle, R. W. Fundamentos do treinamento de força e do condicionamento. Editora Manole: São Paulo, 2010.

Barbosa Filho, A., et al. Práticas esportivas e recreativas em adolecentes com execesso de peso: análise da composição corporal e do desempenho motor. Motriz. 2011; 17 (2), 264-273.

Bittencourt, N. Musculação: uma abordagem tecnológica. Editora Sprint: Rio de Janeiro, 1986.

Bouchard, C., Tremblay, A. Genetic effects in human energy expenditure components. Int J Obes. 1990; 14: 49-58.

Carmo, E. H., Barreto, M. L., Silva, J. R. Changes in the pattern of morbidity and mortality of the brazilian population: challenges for a new century. Epidemiol Serv Saúde. 2003; 6(2): 63-75.

Castro, S. H. D., Mato, H. J. D., Gomes, M. D. B.. Parâmetros antropométricos e síndrome metabólica em diabetes tipo 2. Arquivos Brasileiros de Endocrinologia \& Metabologia. 2006; 50: 450-455.

Dietz, W. H., Gortmaker, S. L. Do we fatten our children at the television set? Obesity and television viewing in children and adolescents. Pediatrics. 1985; 75: $805-12$.

Faigenbaum, A. D., Westcoyy, W. L. Youth strength training: programs for health. Fitness andsport. Editora Human Kinetcs, 2009.

Farias, E. S., et al. Efeito da atividade física programada sobre a composição corporal em escolares adolescentes. J. Pediatr. Rio J. 2009; 85(1): 28-34.

Ferreira, M. G., Valente, J. G., Silva, R. M. V. G., Sichieri, R. Acurácia da circunferência da cintura e da relação cintura/quadril como preditores de dislipidemias em estudo transversal de doadores de sangue de Cuiabá, Mato Grosso, Brasil. Cad. Saúde Pública. 2006; 22(2): 307-314.

Garcia, J. A. D., Incerpi, E. K. Fatores e mecanismos envolvidos na hipertrofia ventricular esquerda e o papel anti-hipertrófico do óxido nítrico. Arquivos Brasileiros de Cardiologia. 2008; 90(6): 443-50.

Gharakhanlou, R., Farzad, B., Agha-Alinejad, H., Steffen, L. M., Bayati, M. Anthropometric Measures as Predictors of Cardiovascular Disease Risk Factors in the Urban Population of Iran. Arq Bras Cardiol. 2012; 98(2): 126-135. 
IBGE - Instituto Brasileiro de Geografia e Estatística. Pesquisa de Orçamentos Familiares 2008-2009: desnutrição cai e peso das crianças brasileiras ultrapassa padrão internacional. 2010. [acesso em 23 fev 2011].

Disponível em:

<http://www.ibge.gov.br/home/presidencia/noticias/noti cia_visualiza.php?id_noticia=1699 HYPERLINK "http://www.ibge.gov.br/home/presidencia/noticias/noti cia_visualiza.php?id_noticia $=1699 \& i d$ pagina $=1 " \&$ HYPERLINK

"http://www.ibge.gov.br/home/presidencia/noticias/noti cia_visualiza.php?id_noticia $=1699 \& i d \_$pagina=1"id_p agina $=1>$

IDF. INTERNATIONAL DIABETES FEDERATION.

The IDF Consensus Worldwide Definition of the Metabolic Syndrome in Childrens and Adolescents. JAMA. 2001; 285(19): 2486-97.

Martinelli, B., et al. Influência do exercício aeróbio na renina de portadores de hipertensão arterial com sobrepeso. Arquivos Brasileiros de Cardiologia. 2010; 95(1): 91-98.

Morris, F., Naughton, G., Gibbs, J., Wark, J. Prospective tem-month exercise intervention in premenarcheal girls: positive effects on bone and lean mass. Journal of Bone and Mineral Research. 1997; 12 : 1453-1462.

Nelson, M. E., et al. Physical activity and public health in older adults: recommendation from the Americam College of Sports Medicine and the American Heart Association. Med Sci Sports Exerc. 2007; 39(8): 1435 1445.

Neutzling, M. B., et al. Overweight and obesity in Brazilian adolescents. Int. J. Obes. 2000; 24(7): 869874.

Ogden, C. L., Carroll, M. D., Flegal, K. M. High body mass index for age among US children and adolescents, 2003-2006. JAMA. 2008; 299(20): 2401-2405.

Porto, M., Nagamine, K. K., Brandão, A. C., Florim, G. S., Pinhel, M. A., Souza E. O., Souza D. R. S.; Resistance Training Program on Body Composition and Muscle Strength in Children with Obesity. R. Bras. Ci. e Mov. 2013; 21 (4): 21-29.

Ruiz, A. T., Ruiz, J. V. T. Aspects practices del treatment de I'obesity infantil. Apunts. 1993; 30: 11734.

Sabia, R. V., Santos, J. E., Ribeiro, R. P. P. Efeito da Atividade Física Associada à Orientação Alimentar em Adolescentes Obesos: comparação entre o exercício aeróbico e anaeróbico. Rev Bras Med Esporte. 2004; 10(5): 349-355.
Silva, D. F., Bianchini, J. A. A., Nardo Junior, N. Tratamento multiprofissional da obesidade e sua sessação em adolecentes: efeitos no perfil hemodinâmicos. Motriz. 2013; 19(1): 195-206.

Schutz, Y. Macronutrients and energy balance in obesity. Metabolism. 1995; 44(3): 7-11.

Sothern, M., Loftin, J., Udall, J., Suskind, R., Ewing, T., Tang, S., Blecker U. Safety, feasibility and efficacy of a resistance training program in preadolescent obese youth. The American Journal of The Medical Sciences. 2000; 319(6): 370-375.

Thomas, N. E., et al. Relationship of fitness, fatness, and coronary-heart-disease risk factors in 12- to 13year-olds. Pediatr. Exerc. Sci. 2007; 19(1): 93-101.

WHO, World Health Organization. Preventing and managing the global epidemic. Report of a WHO Consultation. Geneva. 2000. [acesso em 17 out 2016]. Disponível em

http://whqlibdoc.who.int/trs/who_trs_894.pdf.

Yusef, C. C. W., Sung, R. I. T., So, R. C. H., Lui K, C., Lau, W., Lam, P. K. W. Effects of strength training on body composition and bone mineral content in children who are obese. J Strength Cond Res. 2005; 19: 667672 .

\section{Bruna Wood Almeida Mendes}

Graduada em Educação Física pela Universidade José do Rosário Vellano (UNIFENAS).

\begin{tabular}{l} 
Renata Alves de Lima \\
Graduada em Educação Física pela Universidade \\
José do Rosário Vellano (UNIFENAS). \\
\hline
\end{tabular}

Sildilaine Aparecida Correia Costa

Graduada em Educação Física pela Universidade José do Rosário Vellano (UNIFENAS).

\section{Giuliano Roberto da Silva}

Doutor em Promoção de Saúde pela Universidade de Franca (UNIFRAN).

\section{Gerusa Dias Siqueira Vilela Terra}

Doutora em Promoção de Saúde pela Universidade de Franca (UNIFRAN). Diretora de graduação da Universidade José do Rosário Vellano (UNIFENAS).

Cassiano Merussi Neiva

Livre Docente pela UNESP. Coordenador do 
Laboratório de Metabolismo e Fisiologia do Esforço (MEFE) - UNESP.

\section{Bruno Barbosa Rosa}

Mestre em Promoção de Saúde pela Universidade de Franca (UNIFRAN). 